\title{
Might quantum-induced deviations from the Einstein equations detectably affect gravitational wave propagation?
}

\author{
Adrian $\mathrm{Kent}^{1,2, *}$ \\ ${ }^{1}$ Centre for Quantum Information and Foundations, DAMTP, Centre for Mathematical Sciences, \\ University of Cambridge, Wilberforce Road, Cambridge, CB3 OWA, U.K. \\ ${ }^{2}$ Perimeter Institute for Theoretical Physics, 31 Caroline Street North, Waterloo, ON N2L 2Y5, Canada.
}

(Dated: October 2012 (revised March 2013))

\begin{abstract}
A quantum measurement-like event can produce any of a number of macroscopically distinct results, with corresponding macroscopically distinct gravitational fields, from the same initial state. Hence the probabilistically evolving large-scale structure of space-time is not precisely or even always approximately described by the deterministic Einstein equations.

Since the standard treatment of gravitational wave propagation assumes the validity of the Einstein equations, it is questionable whether we should expect all its predictions to be empirically verified. 'In particular, one might expect the stochasticity of amplified quantum indeterminacy to cause coherent gravitational wave signals to decay faster than standard predictions suggest. This need not imply that the radiated energy flux from gravitational wave sources differs from standard theoretical predictions. An underappreciated bonus of gravitational wave astronomy is that either detecting or failing to detect predicted gravitational wave signals would constrain the form of the semi-classical theory of gravity that we presently lack.
\end{abstract}

\section{INTRODUCTION}

One difficulty theoretical physicists currently face is that, as the subject has grown larger and speculative attempts to address fundamental problems have multiplied, our collective knowledge has become increasingly fragmented. Questions which are at the forefront of the attention of one group of people can be pretty much neglected, or not even recognised, by others. Indeed, even individuals may display a version of this: because our attention is selective and trained, we can end up functioning according to a sort of doublethink, in which we note a problem in one context and neglect it in others. I suspect this is actually much more common than we generally realise.

Here, following a broader programme ([1, 11-14]; see also [8 10] for earlier distinct but related ideas in this area) of trying to test underexplored foundational questions relating quantum theory and gravity, I suggest one area - gravitational wave physics - where this phenomenon may be at work. The problem is this. On the one hand, the standard theory of propagating gravitational waves treats them as perturbations of the Einstein gravitational field equations. On the other hand, there is no way of incorporating unpredictable quantum events into a classical theory of gravity described by the Einstein equations.

This is not only because of small corrections arising from an as yet unknown quantum theory of gravity. If this were the only issue, it might be easier to defend the case that the standard perturbative treatment of gravitational waves is likely to be essentially unaltered by quantum corrections, at least in regions where the grav-

*a.p.a.kent@damtp.cam.ac.uk itational field is not too strong. The more immediate problem is that, whenever a quantum measurement type interaction takes place - whether a deliberate measurement by a human observer or a naturally occurring event — it can produce any of a number of macroscopically distinct results from the same initial state. According to the standard understanding of quantum theory, these measurement outcomes are intrinsically probabilistic. Not only can they not be predicted in advance by quantum theory, but there are very strong reasons [2 5] to believe that no underlying deterministic theory allows us to predict them. These macroscopically distinct outcomes lead to distinct space-times and matter distributions. Each of several possible space-times and matter distributions could thus emerge from the same initial state. If the measurement event is suitably amplified, their differences can be arbitrarily large. Since general relativity is deterministic, it follows that the Einstein equations cannot even approximately describe the large-scale structure of spacetime around measurement events.

If we try to describe the space-time physics in the vicinity of the measurement event by some classical stressenergy tensor and metric, it seems that we need to introduce some stochastic source which creates an unpredictable macroscopic perturbation of the metric and tensor, at some point - or, probably more accurately, in some region - in the vicinity of the event. However, not only are the Einstein equations inconsistent with these perturbations, but moreover we know of no generally valid way of incorporating them into some semi-classical theory coupling the metric to a classical quantity derived from the quantum stress-energy tensor, or by some stochastic modification of the Einstein equations.

Looking at current knowledge without a predefined theoretical agenda, then, all we can really say for sure is that general relativity and quantum theory both work well in their respective domains. However, even charac- 
terizing precisely those domains of confirmed validity is subtler and harder than it first seems, as the comments above illustrate. It is even harder to justify confidence that we now understand all the principles underlying a unified theory. It's not evident that mainstream ideas work, and while it's certainly not evident that comparatively undeveloped alternatives will work either, they do exist.

For example, it is certainly possible to imagine unifications in which both general relativity and quantum theory work as good approximations in their respective domains, and in which gravity is quantized, but nonetheless the structure of space-time is also constrained by additional laws that modify the probability of each spacetime and are defined by intrinsically geometric rules that do not follow from any quantum theory [1]. It's also possible to imagine theories in which gravity is not quantized at all, and the laws of nature define some probability distribution on classical space-times with quantum matter distributions [1].

The general hypothesis that gravity and quantum state collapse could be linked [8 10], via fundamentally nonunitary dynamical laws is also intriguing, even though it too is hard to make into a precise theory.

Also, even if one of the more currently popular approaches to quantum gravity is correct, it is very unclear how to derive from it a phenomenological higher-level theory that couples microscopic quantum matter with macroscopic events in space-time, or precisely what features such a theory would have.

To reiterate, the point here is not to advocate these comparatively undeveloped alternative ideas, but simply to underline that unifying gravity and quantum theory is an open subject and there are many un(der)explored possibilities. Even in macroscopic regimes with weak gravitational fields, we simply do not have a theory of matter and space-time good enough to fit all observable data. This problem occupies a peculiar status in modern physics: it cannot be denied that the problem is there, but yet most discussions of quantum theory and gravity ignore it. The gap in the literature is so glaring that one almost gets the impression that it is somehow seen as scientifically unsophisticated to look for a theory - even a provisional phenomenological theory - that actually fits the available empirical data.

To sum up: the Einstein equations do not actually correctly describe the large-scale structure of space-time and finding equations that do is an open question. It thus doesn't seem so obvious that the long-range propagation of gravitational waves is necessarily correctly described by considering them as perturbations of the Einstein equations.

The rest of this paper attempts to flesh out this point mostly by conceptual, rather than mathematical, argument. In mitigation, I would stress again that we know nothing for certain about unifying quantum theory and gravity, and the world possibly already has more than enough mathematically rigorous, but conceptually prob- lematic, and quite likely ultimately irrelevant, calculations based on speculative mathematical ideas about how to solve the problem.

Our ultimate aim, of course, is to test precise mathematical theories against quantitative experimental data, but just at the moment we need new ideas about where to look. It seems to me there are strong reasons to try a different style of scientific reasoning: namely, to look at interesting experiments and observations where we don't yet know for sure what theory predicts but can - now, or soon - get an empirical answer, and to ask whether there is any semi-plausible phenomenological model or intuition that might contradict the standard expectation (if there is one). Either we verify with certainty interesting features of gravitational physics that were previously either ignored completely or assumed without compelling evidence, or (even better) we learn something new and surprising.

\section{PRIOR TESTS OF PROBABILISTIC SEMI-CLASSICAL GRAVITY}

\section{A. The Page-Geilker Experiment}

The fundamental problem in constructing semiclassical gravity theories was illustrated by a very simple experiment carried out some time ago by Page and Geilker [6]. Page and Geilker's aim was to refute conclusively the hypothesis that a classical gravitational field couples to quantum matter via the semiclassical Einstein equations

$$
G_{\mu \nu}=8 \pi\left\langle\psi\left|T_{\mu \nu}\right| \psi\right\rangle,
$$

where $G_{\mu \nu}$ is the Einstein tensor and $T_{\mu \nu}$ the quantum stress-energy operator. Their hypothesis presupposes an Everettian interpretation of quantum theory, so that the matter field quantum state, $|\psi\rangle$, is supposed to evolve unitarily without collapse.

As Page and Geilker noted, this hypothesis already seemed intrinsically unlikely (perhaps even incredible) before they carried out their experiment, since it is hard to imagine how it could lead to a cosmological theory which accounts for our observation of gravitational fields generally consistent with those predicted by the Einstein equations from the observed positions of astronomical bodies. Page and Geilker's motivation for their experiment is thus seriously questionable. Perhaps, though, one could imagine some form of theory in which a classical gravitational field couples to the expectation value of quantum matter, while the quantum matter state over time collapses towards definite values for $T_{\mu \nu}$. In any case, it still seems good to have conclusive experimental confirmation even of very solidly based theoretical expectations when, as here, we have no complete theory.

The experiment proceeded by counting the number of decays detected from a radioactive source in a given time interval, and then manually placing large $(\approx 1.5 \mathrm{~kg})$ lead 
balls in one of two configurations, with the choice of configuration depending on the decay count. A Cavendish torsion balance, sensitive enough to distinguish between the two configurations, was used to measure the local gravitational field. To good approximation, Eq. (1) predicts that in each run the experimenter should (whichever of the two configurations they place the masses in) observe a gravitational field defined by the weighted average of the fields corresponding to the two possible configurations. As most expected, the results were consistent with the hypothesis that the gravitational field is determined by the configuration of the masses chosen in any given run of the experiment, and inconsistent with Eq. (1).

We can flesh out the implications of the experiment or, to capture the historical flow of ideas better, perhaps one should say the implications of the generally held prior intuition that its results would be those which were in fact observed - by looking at three possible solutions to the Einstein equations.

First, consider the classical metric and matter fields, which we denote by $\left(g_{\mu \nu}^{0}, \phi^{0}\right)$, in the neighbourhood of some spacelike hypersurface $S$ before the point at which a Page-Geilker experiment is carried out. By "the classical metric and matter fields", we mean here the fields that would ordinarily be defined by someone trying to model the local space-time neighbourhood using general relativity - for instance, an engineer, trying to predict how small lumps of matter will evolve, and doing so as precisely as is possible without taking quantum theory into account. Let us suppose there is some way of fitting these data to a solution of the Einstein equations, using some well-defined and natural recipe (not necessarily Eq. (1)) to obtain a classical stress-energy tensor from the quantum matter field, and denote the corresponding spacetime by $\Sigma_{0}$.

Now consider the classical metric and matter fields, $\left(g_{\mu \nu}^{1}, \phi^{1}\right)$ and $\left(g_{\mu \nu}^{2}, \phi^{2}\right)$, in the neighbourhood of a spacelike hypersurface $S^{\prime}$ after the point at which a PageGeilker experiment is carried out. Let us suppose these data can also be fitted to solutions of the Einstein equations, using the same recipe for a classical stress-energy tensor as before, and denote the corresponding spacetimes by $\Sigma_{1}$ and $\Sigma_{2}$.

Since $\Sigma_{1}$ and $\Sigma_{2}$ describe macroscopically distinct matter configurations on $S^{\prime}$, they are not identical, and so cannot both be identical to $\Sigma_{0}$. In fact, since neither of them is preferred in any way, one would not generally expect either of them to be identical to $\Sigma_{0}$, assuming that the recipe used to obtain the stress-energy tensors depends in any natural way on the relevant fields. (Obviously, if completely arbitrary recipes are allowed, one could contrive things so that one of them equals $\Sigma_{0}$. For instance, one could define the recipe for obtaining $\Sigma_{0}$ to involve first studying the possible experimental outcomes, then constructing $\Sigma_{1}$ and $\Sigma_{2}$, and then simply setting $\Sigma_{0}$ to be equal to one of them.)

To sum up, then, if we can find a way of describing the data before and after the experiment by piecewise continuous solutions of the classical Einstein equations, they will generally be solutions that form part of different spacetimes. In this description (if there is indeed such a description), it is as though some sort of stochastic jump takes place, starting from one solution, and arriving at one of two alternative solutions, both distinct from the original.

Is there such a description? Can the spacetime we actually observe be described by piecewise continuous solutions of the Einstein equations? I don't think we know for sure: some sort of smoothing could take place in the vicinity of Page-Geilker experiments, for instance. But it seems to be generally tacitly assumed - and it seems to be necessary to assume, in order to explain experimental data - that this is at least approximately the case. For, on the one hand, if it were not the case that large regions of spacetime are well described by a solution to the Einstein equations, we would not be able to account theoretically for any of the confirmed predictions of general relativity. On the other hand, as we have just argued, the sort of macroscopic indeterminacy exhibited by the Page-Geilker experiment implies that spacetime cannot be described globally by a single solution of the Einstein equations.

\section{B. Probing gravitational non-locality and the Salart et al. experiment}

A more recent proposal [12] with some related motivations was to look for direct evidence of violations of Bell's local causality in the gravitational field. Recall that Bell experiments (modulo loopholes) show that any hidden variable theory underlying quantum theory must violate Bell's condition of local causality. Since non-local hidden variable theories are theoretically uncompelling and difficult to reconcile with relativity, this gives much stronger evidence that the outcomes of quantum experiments are indeed inherently unpredictable. The evidence that the classical gravitational field evolves probabilistically, though, is less direct. While the Page-Geilker experiment appears to confirm that the gravitational field evolves probabilistically, one could still imagine an underlying deterministic semi-classical gravity theory, sensitive to microscopic variables, that predicts each observed evolution, in the same way that de Broglie-Bohm theory and other deterministic hidden variable theories reproduce the predictions of quantum theory. We would like to be able to argue directly that any deterministic phenomenological theory of gravity must be non-local. Since a non-local gravity theory would be very hard to reconcile with either special or general relativity, this would be a compelling reason for abandoning all hope for deterministic semi-classical theories.

Another strong motivation for verifying this point directly is that gravitational collapse models highlight another possible loophole in the interpretation of all Bell experiments to date. This "collapse locality loophole" 
[11] arises because collapse models suggest that a definite measurement outcome occurs only after macroscopic amplification to a particular scale (which depends on the parameters of the collapse model). If this is correct, to exclude locally causal explanations we need Bell experiments that ensure that collapses, and thus definite measurement outcomes, take place in spacelike separated regions in the two wings. No Bell experiment to date ensures this for the full range of collapse model parameters consistent with known experiment [11].

This motivation is further reinforced by the observation [11] that there are ways in which a consistent theory combining quantum theory and gravity could conceivably produce the outcomes observed in all Bell experiments to date and nonetheless allow only locally causal evolutions of the metric. Of course, models incorporating these ideas have highly non-standard properties, and may be theoretically problematic as well as ad hoc. Still, as with the Page-Geilker experiment, clear experimental data would be much preferable to strong theoretical intuitions and arguments.

A beautiful experiment investigating this possibility was carried out by Salart et al. [13], showing that the local causality loophole can indeed be closed at least for gravitational collapse models whose collapse times and scales agree with theoretically motivated estimates proposed by Penrose [8] and Diosi [9]. Further more conclusive experiments have been proposed [14], with the ultimate aim of directly measuring non-locally correlated gravitational fields in such a way that these measurements are themselves completed in space-like separated regions.

\section{NATURAL MEASUREMENT-LIKE EVENTS}

Of course, quantum measurement-like events with macroscopically distinct consequences take place without any artificial help. On Earth, fissioned particles and cosmic rays leave tracks in mica; frogs can respond to the stimulus of a single photon; a single gamma ray or charged particle can trigger a cancer; the bouncing of photons and cosmic rays off dust particles must from time to time determine the formation or otherwise of a particular macroscopic clumping. Each of these outcomes is effectively a quantum measurement of the position of a particle whose wave function was previously delocalised. On the cosmological scale, quantum fluctuations are believed to have seeded the instabilities that led to galaxy formation.

It would be very interesting indeed to try to quantify the degree to which quantum noise, bubbling up from the microscopic realm, affects the predictability of the macroscopic world in general, and in particular to characterise the degree and type of the resulting deviations from Einstein's equations. This project is beyond our present scope, though.

For the purposes of the present discussion, we need only take the point that these deviations occur naturally, and presumably have been doing so since very early cosmological times. This is why the outcome of the PageGeilker experiment was generally (perhaps even universally) anticipated. In other words, while the Page-Geilker experiment is a good illustration of the point that our observed space-time deviated from Einstein's equations, we do not actually need to appeal to it to make that point.

\section{QUANTUM GRAVITY: RESOLUTION OR DISTRACTION?}

According to one school of thought, at this point in the discussion one should throw up one's hands, regret the fact that we don't yet have a quantum theory of gravity, and accept that we can't productively advance the discussion further without one.

It seems to me far from evident that we should heed this counsel of despair. I can see two reasons for optimism.

First, we might not actually need a quantum theory of gravity at all. Second, even if we do, it ought to imply some effective phenomenological theory of classical gravity which incorporates stochastic fluctuations into general relativity. In the first case, we might hope for some fundamental theory which incorporates stochastic fluctuations into general relativity; alternatively, we might hope for a theory based on different principles, which again should imply an effective phenomenological theory of classical gravity of the type just mentioned.

In all these cases, it is reasonable to try to explore how a classical gravity theory with stochastic fluctuations might be probed experimentally. But we don't have such a theory. Perhaps the best hope, then, is that experiment might guide us to the right theory, if we can at least identify what to look for experimentally.

The following two sub-sections flesh out these arguments in more detail.

\section{A. Do we need quantum gravity to explain the Page-Geilker experiment?}

Embarrassingly, our best theory of gravity, general relativity, has no way of consistently incorporating the results of macroscopically amplified quantum measurement events, whether they occur naturally or are created artificially as in a Page-Geilker experiment. The current conventional wisdom suggests that this embarrassment stems from our failure to devise a consistent quantum theory of gravity.

There is — the standard argument runs - no fully consistent way to couple a classical gravitational field to quantum matter fields: the gravitational field also needs to be quantised. We would expect — the argument proceeds to suggest - that in a full quantum theory of gravity, the gravitational field would evolve so as 
to be (to very good approximation) correlated with the matter fields in any given branch of the universal wave function. In particular, we would expect a full quantum theory of gravity to predict the observed outcome of the Page-Geilker experiment: this is why Page and Geilker provocatively titled their paper "Indirect Evidence for Quantum Gravity". More generally, we would expect a full quantum theory of gravity to predict that the gravitational field should be correlated with the positions of astronomical bodies in the way we observe.

Of course, this argument begs several key questions. Aside from the problems of principle in unifying quantum theory and gravity, discussed above, there is the problem of making scientific sense of purely unitary quantum theory. We do not have, despite nearly fifty years' of effort, any clearly consistent and logically compelling account of how Everett's original intuition might be fleshed out into a clearly and carefully justified interpretation of a unitarily evolving universal wave function. (State-of-the-art reports and assessments of recent attempts can be found in Ref. [7].)

\section{B. Probing an effective theory derivable from quantum gravity}

What if some version of quantum gravity is correct, though? Suppose, for example, we find some rigorously defined way of carrying out path integrals over gravitational and matter field configurations, and find some evidence that it gives correct answers. In order to understand large-scale gravitational physics, we would still need some (presumably) phenomenological effective theory, derived from our fundamental quantum gravity theory, which characterises the quasiclassical behaviour of matter and gravity that we actually observe. (In Gell-Mann and Hartle's terminology [15], we would need some way of characterising our own quasiclassical domain within this hypothetical quantum gravitational or quantum cosmological theory.)

In particular, this higher-order theory would need to be consistent with the Page-Geilker experiment and with the observed correlations of gravitational fields and astronomical bodies. It thus seems a reasonable conjecture - suggested by observational evidence, and contradicted by nothing we know about quantum gravity that we would end up with some sort of stochastically modified version of general relativity, albeit in this case as a derived effective theory rather than a fundamental theory. If so, one might make the further reasonablelooking guess that the propagation of gravitational waves is approximately described by considering them as perturbations of the gravitational field within this higherorder quasiclassical theory

\section{WHAT HAPPENS TO GRAVITATIONAL WAVES IN A STOCHASTIC MODIFICATION OF GENERAL RELATIVITY?}

Without knowing the details, one can only guess. So, without further ado, I shall. A plausible guess, it seems to me, is that stochastic fluctuations break up the coherence of propagating waves. It is difficult to hear someone shouting in a high wind, not only because the noise of the wind drowns out the propagating sound wave, but also because the turbulence causes its amplitude to decay faster than in still air.

If the level of stochastic fluctuations is constant throughout a region in which a wave propagates, the simplest guess would be that the wave amplitude decays by a factor exponential in the region length, in addition to the normal approximately inverse square law decay. Without knowing the theory, one can't estimate the value of the exponential constant - but if this guess is right, and if gravitational wave astronomy turns out nonetheless to be viable, one might be able to estimate it from observational data, and thereby get quantitative data characterising an important feature of the relation between quantum theory and gravity.

This raises the possibility that the stochastically induced decay of gravitational waves could conceivably prevent gravitational wave astronomy from being viable with presently envisaged gravitational wave detectors. If so, of course, gravitational wave astronomy's loss would be gravitational theory's gain.

\section{WHAT ABOUT THE BINARY PULSAR OBSERVATIONS?}

If one suggests the possibility that the standard account of gravitational wave physics might not be correct, one has to deal with the counter-argument that observations of binary pulsars [16] have already confirmed the standard account to a very impressive degree of precision. This counter-argument has no force against the speculations considered here, though. The suggestion is not that binary pulsars do not emit gravitational waves, and thereby lose energy, as standard theory predicts. The suggestion is, rather, that the gravitational waves lose coherence, and thus decay faster than expected, as they propagate through space, and hence in particular that gravitational wave signals reaching Earth might be weaker than anticipated. Careful observation of a drum vibrating in the distance would reveal that it is losing energy by radiating sound waves; nonetheless, the sound of the drum will not propagate as far in a strong wind. There is no evident inconsistency here. 


\section{COMPARING QUASICLASSICAL GRAVITY AND QUASICLASSICAL ELECTRODYNAMICS}

To what extent are the problems we raise about our understanding of quasiclassical physics specific to gravity? In particular, are there any reasons to think that classical gravitational waves might behave differently from classical electromagnetic waves?

In considering these questions, it's helpful first to consider quasiclassical electrodynamics in Minkowski space. Clearly, some of the points made above apply. In particular, we can carry out Bell experiments and ensure that, on each wing, a source of electromagnetic waves behaves differently depending on the measurement setting and outcome on that wing, and that the measurement settings themselves are locally determined by random quantum events. For example, a charged sphere could be move in any of four different ways, depending on the two measurement choices and two possible outcomes, and the measurement choices could be determined, just before the measurements are made, using bits produced by quantum random number generators. Since the outcomes of Bell experiments are nonlocally correlated, we expect this to produce nonlocal correlations in the electromagnetic fields propagating from the regions of the two measurements.

Now, this probably has not been directly tested in experiments to date, and I am not sure we can in principle rigorously exclude models (with very counterintuitive features) that agree with experiments to date but predict that nonlocal correlations of classical electromagnetic fields cannot in fact be observed. Of course, this would be a very surprising outcome indeed. We ignore the possibility here, since our aim is to understand whether one might have possible reasons to look for unexpected behaviour in quasiclassical gravitational physics even if there are no analogous surprises in quasiclassical electrodynamics.

Assuming, then, that nonlocal correlations can be created in macroscopic electromagnetic fields, it follows that quasiclassical electrodynamics in the real world cannot be described by an underlying local deterministic model. Note, though, that the nonlocalities we introduced arise entirely from nonlocal correlations in the motion of sources. Given a description of the motion of each source, we can calculate the subsequent behaviour of the electromagnetic fields it generates. Since electrodynamics is a linear theory, we can obtain a complete solution by superposing the contributions from the various sources. This gives a strategy for building a phenomenological model of quasiclassical electrodynamics in the presence of quantum unpredictability and nonlocality: first apply the predictions of quantum theory to give a model of additional stochastic (and nonlocally correlated) forces acting on the sources, and then solve to obtain the fields. Adding forces that alter the motion of the sources does not affect charge conservation, so in such a model we still have $\partial_{\mu} J_{\mu}=0$.

It would be wrong to suggest this gives a rigorous understanding of the relationship between quantum and quasiclassical electrodynamics in Minkowski space. We do not even have a completely rigorous definition of quantum electrodynamics as a non-trivial theory. Nor do we have a precise general prescription for how to obtain quasiclassical equations of motion from quantum theory, either for electrodynamics or for any other physically relevant theory. However, we do at least have an ansatz for dealing with the quasiclassical consequences of quantum experiments with unpredictable and nonlocally correlated outcomes, and this ansatz does not violate the conservation laws necessary for a consistent solution of the electrodynamic equations.

Now compare the situation when we try to model an analogous experiment in which the measurement choices and outcomes of Bell experiments determine the motion of massive objects on each wing, with the measurement choices again locally determined by quantum random number generators. As noted earlier, we cannot model the quasiclassical physics by extrapolating the predictions of general relativity from data on a spacelike hypersurface before the Bell experiment, since general relativity is deterministic and the Bell data are not. So far the analogy with electrodynamics holds, since electrodynamics is also deterministic. However, we run into further problems in this case. To define any consistent solution of the Einstein equations, we need the local conservation of stress-energy, $D_{\nu} T^{\mu \nu}=0$. We know of no generally covariant quasiclassical model of the possible outcomes of quantum measurement-like processes that preserves stress-energy and is consistent with general relativity where quantum effects are negligible. (Indeed, even non-relativistic dynamical collapse models [17, 18], which might be the best guesses at phenomenological descriptions of the quasiclassical physics emerging from measurement interactions, violate conservation of energy.) Without such a model, it seems our best description of quasiclassical gravitational physics would be by models which generally obey the Einstein equations but have singularities or discontinuities at or in the vicinity of quantum measurement events. And if that were the best possible description, the standard classical derivation of gravitational wave propagation would break down in these regions.

To be sure, there are further uncertainties here. If these discontinuities are physically real, should we expect them to affect the propagation of electromagnetic radiation in the same way as they affect the propagation of gravitational waves? If so, of course, any effect is likelier to be evident in standard (electromagnetic wave observation) astronomy than in gravitational wave astronomy, and the absence of any observed effect to date is a strong constraint. On the other hand, we have a quantum theory of electromagnetism and no quantum theory of gravity. And, if there is a quantum theory of gravity from which quasiclassical solutions obeying Ein- 
stein's equations with discontinuities emerges, we have no clear reason to think that coherent beams of gravitons and photons should scatter similarly from the discontinuities - indeed one might guess that gravitons are more directly affected than photons by a discontinuity in the classical field generated by gravitons.

Some may nonetheless hold the intuition that we should expect exactly the same effects in quasiclassical gravity and quasiclassical electrodynamics. The points made here do not refute this possibility, but they do give significant reasons to query it.

\section{SUMMARY}

In this paper, we raised a possibility that does not seem to have been considered: that stochastic corrections to the Einstein equations dissipate gravitational waves. Such stochastic corrections could either arise directly from a fundamental theory or as a phenomenological effect resulting from quantum gravity (or some other presently unknown type of theory). Either way, our guess at their effect on gravitational wave propaga- tion is not provable given the present state of theoretical understanding. But is it obviously wrong, or totally implausible? If, as we suggest, not, it seems a possibility to be kept in mind if and when gravitational wave astronomy produces data, null or otherwise. We hope too that the questions raised here may encourage more attention to be focussed on the problem of finding realistic quasiclassical descriptions of gravitational physics in the presence of quantum measurements, through Bell experiments and otherwise.

\section{ACKNOWLEDGEMENTS}

This work was partially supported by a Leverhulme Research Fellowship, a grant from the John Templeton Foundation, and by Perimeter Institute for Theoretical Physics. Research at Perimeter Institute is supported by the Government of Canada through Industry Canada and by the Province of Ontario through the Ministry of Research and Innovation.
[1] A. Kent, Phys. Rev. A 87, 022105 (2013).

[2] J. S. Bell, Speakable and Unspeakable in Quantum Mechanics (Cambridge University Press, Cambridge, 1987).

[3] J. Clauser, M. Horne, A. Shimony and R. Holt, Phys. Rev. Lett. 23, 880 (1969).

[4] Colbeck, R. and Renner, R., Bulletin of the American Physical Society, 56 (2011).

[5] Pusey, M.F. and Barrett, J. and Rudolph, T., Nature Phys. 8, 476 (2012).

[6] D. Page and C. Geilker, Phys. Rev. Lett. 47979 (1981).

[7] S. Saunders, J. Barrett, A. Kent, and D. Wallace. Many worlds?: Everett, quantum theory, and reality. Oxford University Press, 2010.

[8] R. Penrose, The Emperor's New Mind (Oxford University Press, Oxford, 1999) and refs therein.

[9] L. Diosi, Phys. Rev. A40 1165 (1989).

[10] P. Pearle and E. Squires, quant-ph/9503019

[11] A. Kent. Causal quantum theory and the collapse locality loophole. Physical Review A, 72(1):012107, 2005.

[12] A. Kent. A proposed test of the local causality of spacetime. Quantum Reality, Relativistic Causality, and Closing the Epistemic Circle, pages 369-378, 2009.

[13] D. Salart, A. Baas, JAW Van Houwelingen, N. Gisin, and H. Zbinden. Spacelike separation in a Bell test assuming gravitationally induced collapses. Physical review letters,
100(22):220404, 2008.

[14] Fundamental quantum optics experiments conceivable with satellites - reaching relativistic distances and velocities D. Rideout et al., Classical and Quantum Gravity 29224011 (2012).

[15] M. Gell-Mann and J.B. Hartle in Complexity, Entropy, and the Physics of Information, SFI Studies in the Sciences of Complexity, Vol. VIII, ed. by W. Zurek, Addison Wesley, Reading (1990); M. Gell-Mann and J.B. Hartle in Proceedings of the NATO Workshop on the Physical Origins of Time Asymmetry, Mazagón, Spain, September 30-October 4, 1991 ed. by J. Halliwell, J. Pérez-Mercader, and W. Zurek, Cambridge University Press, Cambridge (1994); M. Gell-Mann and J.B. Hartle, Phys. Rev. D 47 3345 (1993).

[16] J. Taylor, L. Fowler, and J. Weisberg, Nature 277, 437 (1979).

[17] G.C. Ghirardi, A. Rimini, and T. Weber. Unified dynamics for microscopic and macroscopic systems. Physical Review D, 34(2):470, 1986.

[18] Gian Carlo Ghirardi, Philip Pearle, and Alberto Rimini. Markov processes in Hilbert space and continuous spontaneous localization of systems of identical particles. Phys. Rev. A, 42:78-89, Jul 1990. 